\title{
Ethoxysulfuron no controle de plantas daninhas na cultura do feijoeiro comum
}

\section{Ethoxysulfuron on weed control in common bean crop}

\author{
Fortunato Pagnoncelli ${ }^{*}$, Ribas Antonio Vidal ${ }^{2}$, Michelangelo Muzell Trezzi ${ }^{1}$, \\ Sorhaila Camila Batistel ${ }^{3}$, Renata Caroline Gobetti ${ }^{1}$, Bruna Mandryk Cavalheiro ${ }^{1}$, Matheus Viecelli ${ }^{1}$
}

Resumo - É necessário identificar novos herbicidas com amplo espectro de controle de plantas infestantes para a cultura do feijão e, que possuam margem de segurança adequada para a cultura. Os objetivos deste trabalho foram, avaliar o potencial uso do herbicida ethoxysulfuron para o controle de plantas infestantes em meio a cultura do feijão, assim como a tolerância da cultivar de feijão IAC Imperador a este herbicida. Um experimento foi conduzido a campo em delineamento de blocos ao acaso com parcelas subdivididas. Nas parcelas principais foram alocadas as doses de ethoxysulfuron $\left(0 ; 10,4 ; 20,8 ; 41,6 ; 62,5\right.$ e 83,3 $\left.\mathrm{g} \mathrm{ha}^{-1}\right)$ e nas sub parcelas três cultivares de soja (AMS Tibagi, Dow 5D634 e Nidera 5909) + as plantas infestantes (Bidens spp., Ipomoea spp.). Adicionalmente foi incluído um tratamento extra sem infestantes e sem aplicação do herbicida. Ethoxysulfuron controlou as plantas de Ipomoea spp. e soja, mas não as de Bidens spp. Constatou-se tolerância diferencial entre as cultivares de soja avaliadas, em que a cultivar AMS Tibagi indica ser ligeiramente mais tolerante que as demais cultivares. Doses de até 20,8 $\mathrm{g} \mathrm{ha}^{-1}$ são suficientes para controlar satisfatoriamente plantas de Ipomoea spp. e soja, no entanto, não as de Bidens spp. O herbicida proporcionou incremento no rendimento de grãos de feijão, todavia, não foi suficiente para alcançar o rendimento da testemunha extra. A cultivar de feijão IAC Imperador demostrou tolerância moderada ao ethoxysulfuron, além disso, as plantas tendem a se recuperar rapidamente da injúria do herbicida. Palavras-chave: Bidens spp., herbicidas inibidores da ALS, Ipomoea spp., Phaseolus vulgaris, soja

Abstract - It is necessary to identify new herbicides with a wide spectrum of weed control for bean crops, and have an adequate security margin for this crop. The main objectives of this work were to evaluate the potential use of ethoxysulfuron herbicide on controlling weeds and three soybean cultivars between bean crop, as the tolerance of IAC Imperador bean cultivar to this herbicide. An experiment was conducted on field with a split-plot design. At the whole plot were allocated the doses of ethoxysulfuron $\left(0 ; 10.4 ; 20.8 ; 41.6 ; 62.5\right.$; and $\left.83.3 \mathrm{~g} \mathrm{ha}^{-1}\right)$ and, at the subplots, the three soybean cultivars (AMS Tibagi, Dow 5D634 e Nidera 5909) were allocated with weeds (Bidens spp., Ipomoea spp.). Additionally, it was included an extra treatment without weeds and herbicide application. The Ethoxysulfuron controlled Ipomoea spp. and soybean plants, but didn't control Bidens spp. It was found a differential tolerance between the soybean cultivars, and the AMS Tibagi cultivar showed to be slightly tolerant. Doses up to $20.8 \mathrm{~g} \mathrm{ha}^{-1}$ are sufficient to satisfactorily control Ipomoea spp. and soybean plants, but not for Bidens

Recebido: Junho 05, 2017. Aceito: Outubro 20, 2017.

${ }^{1}$ Universidade Tecnológica Federal do Paraná - UTFPR, Departamento de Agronomia, Via do Conhecimento, km 1, CEP 85503-390, Pato Branco, PR, Brasil. E-mail: fpagnoncelli@outlook.com; trezzim@gmail.com; renata_gobetti@hotmail.com; bru.mandryk@hotmail.com; mviecelli@gmail.com

${ }^{2}$ Universidade Federal do Rio Grande do Sul - UFRGS, Departamento de Plantas de Lavoura, Porto Alegre, RS, Brasil. E-mail: ribas.vidal@gmail.com

${ }^{3}$ Universidade Federal de Santa Maria - UFSM, Departamento de Fitotecnia, Santa Maria, RS, Brasil.

E-mail: sc.batistel@hotmail.com 
spp. The herbicide provided an increment of bean yield, however, it wasn't enough to achieve the extra treatment yield. The IAC Imperador bean cultivar showed moderate tolerance to ethoxysulfuron, and its plants can recover quickly after any injurie.

Keywords: Bidens spp., ALS inhibitors herbicides, Ipomoea spp., Phaseolus vulgaris, soybean

\section{Introdução}

O feijoeiro (Phaseolus vulgaris L.) é uma cultura amplamente difundida em todo o território nacional, devido a sua importância econômica e alimentar. Em nível de campo, um dos principais fatores que limita o alcance do potencial de rendimento da cultura é a presença de plantas infestantes.

A redução no rendimento de grãos de feijão pode variar de 35 a $80 \%$, quando a cultura convive por todo seu ciclo com estas plantas (Kozlowski et al., 2002; Borchartt et al., 2011). Logo, torna-se evidente a importância da adoção de medidas profiláticas que reduzam a incidência e/ou a capacidade competitiva das plantas infestantes em áreas cultivadas com feijoeiro. No que se refere a intensidade da interferência, o grau de semelhança morfológica entre as espécies é um dos principais fatores envolvidos (Wassmuth et al., 2009), sendo que, quanto maior a semelhança entre as espécies, maior será a competitividade por recursos do meio. Todavia, cada espécie possui características próprias, que se destacam de acordo com o ambiente em que estão presentes (Radosevich et al., 2007).

O manejo químico de plantas infestantes na cultura do feijão é uma prática amplamente difundida, devido a sua simplicidade e agilidade em relação aos métodos de manejo físico e mecânico. No Brasil, para o controle de plantas infestantes na cultura do feijão, são registrados 27 herbicidas pertencentes a nove mecanismos de ação, o que pode ser considerado baixo se comparado com a cultura da soja, que possui 55 herbicidas divididos em 11 mecanismos de ação (Brasil, 2016). Contudo, dentre os 27 herbicidas, apenas cinco possuem registro para o controle de infestantes dicotiledôneas em pós-emergência da cultura. A baixa disponibilidade pode ocorrer devido a maior sensibilidade da cultura a herbicidas, quando comparada à soja.

A eficiência do herbicida para o manejo de plantas daninhas é determinada pela capacidade do produto retardar ou suprimir o desenvolvimento da infestante, proporcionando o menor prejuízo possível para a planta cultivada. Deste modo, é importante destacar o conhecimento da sensibilidade de diferentes espécies infestantes a herbicidas. Mesmo em uma população considerada sensível, o nível de controle torna-se dependente da dose do herbicida, estágio de aplicação, tecnologia de aplicação e das condições do ambiente (Oliveira Junior e Inoue, 2011). Quando o herbicida perde eficiência em função de alguma destas práticas inadequadas, a infestante torna-se competitiva com a cultura.

O herbicida ethoxysulfuron pertencente ao grupo químico das sulfonilureias, possui registro para o controle de plantas infestantes nas culturas da cana de açúcar e arroz (Rodrigues e Almeida, 2011). O herbicida demostra ser seletivo a cultura do feijão, além disso, possui potencial uso para o controle de plantas voluntárias de soja em meio a cultura (Assis et al., 2014). Devido à baixa disponibilidade de herbicidas recomendados para cultura do feijão, é necessário a identificação de novos herbicidas, com amplo espectro no controle de infestantes e que promovam margem de segurança adequada para que a cultura mantenha sua habilidade competitiva e produtividade de grãos.

Os objetivos deste trabalho foram avaliar o potencial uso do ethoxysulfuron para o controle de plantas infestantes em meio a cultura do feijão, bem como a tolerância da cultivar de feijoeiro IAC Imperador a este herbicida.

\section{Material e métodos}

O experimento foi conduzido a campo no município de Pato Branco $\left(26^{\circ} 17^{\prime} \mathrm{S}\right.$ e $52^{\circ} 69^{\prime} \mathrm{O}$ e $760 \mathrm{~m}$ de altitude) entre os meses janeiro a abril de 2015. As características físicas e químicas do solo são apresentadas na Tabela 1. Os dados de 
precipitação e temperatura são demostrados na Figura 1.

O experimento foi conduzido em blocos ao acaso com parcelas subdivididas com quatro repetições. Nas parcelas principais foram alocadas seis doses de ethoxysulfuron (Gladium, 600 g i.a. kg, Bayer) $\left(0 ; 10,4 ; 20,8 ; 41,6 ; 62,5\right.$ e $\left.83,3 \mathrm{~g} \mathrm{ha}^{-1}\right)$ e as sub parcelas foram ocupadas por 3 cultivares de soja (AMS Tibagi, Dow 5D634 e Nidera 5909), as quais foram consideradas como plantas competidoras

Tabela 1. Distribuição granulométrica e atributos químicos de Latossolo Vermelho Distroférrico.

\begin{tabular}{cccc}
$\begin{array}{c}\text { Distribuição } \\
\text { granulométrica }\end{array}$ & \multirow{2}{*}{\begin{tabular}{c} 
Atributos químicos \\
\cline { 1 - 1 } Componente
\end{tabular}} & Componente \\
\cline { 1 - 3 } Argila & 55,7 & $\mathrm{MO}^{1 /}$ & 4,9 \\
Areia & 3,0 & $\mathrm{P}_{2} \mathrm{O}_{5}{ }^{2 /}$ & 14,3 \\
Silte & 41,3 & $\mathrm{~K}_{2} \mathrm{O}^{3 /}$ & 0,70 \\
& & $\mathrm{CTC}^{4 /}$ & 17,6 \\
& & $\mathrm{pH}^{5 /}$ & 5,6 \\
& & $\mathrm{H}+\mathrm{Al}^{6 /}$ & 5,3 \\
\hline
\end{tabular}

1/ Matéria orgânica $\left(\mathrm{g} \mathrm{dm}^{-3}\right)$; $^{2 /}$ Fósforo $\left(\mathrm{mg} \mathrm{dm}{ }^{-3}\right)$;

3/ Potássio $\left(\mathrm{cmol}_{\mathrm{c}} \mathrm{dm}^{-3}\right)$; 4/ Capacidade de troca de cátions; ${ }^{5 /} \mathrm{pH}$ do solo; ${ }^{6 /}$ Acidez trocável $\left(\mathrm{cmol}_{\mathrm{c}} \mathrm{dm}^{-3}\right)$. (simulando infestação composta por plantas voluntárias). Adicionalmente, foi alocado um tratamento extra, sem aplicação de ethoxysulfuron e livre da presença de infestantes. As parcelas principais possuíam $9 \times 2 \mathrm{~m}$, enquanto que as sub parcelas $3 \times 2 \mathrm{~m}$.

A área estava sob pousio e antes da semeadura do feijoeiro foi realizada uma roçada seguida por gradagem, a fim de estimular a emergência de plantas infestantes no local. Constatou-se predominância das espécies pertencentes aos gêneros Bidens spp. e Ipomoea spp., e por este motivo as demais infestantes foram eliminadas manualmente das parcelas com o intuito de facilitar as avaliações e a análise dos resultados. A semeadura das cultivares de soja foi realizada manualmente a lanço, logo após o plantio do feijoeiro, distribuindo-se aproximadamente 40 sementes por $\mathrm{m}^{2}$.

A cultivar de feijoeiro utilizada foi a IAC Imperador, de ciclo de desenvolvimento precoce (70 a 75 dias) e porte semi erreto (Chiorato et al., 2012). A densidade de planta utilizadas foi de 230 mil plantas ha ${ }^{-1}$. A semeadura foi mecanizada, com uma semeadora adubadora. A adubação utilizada

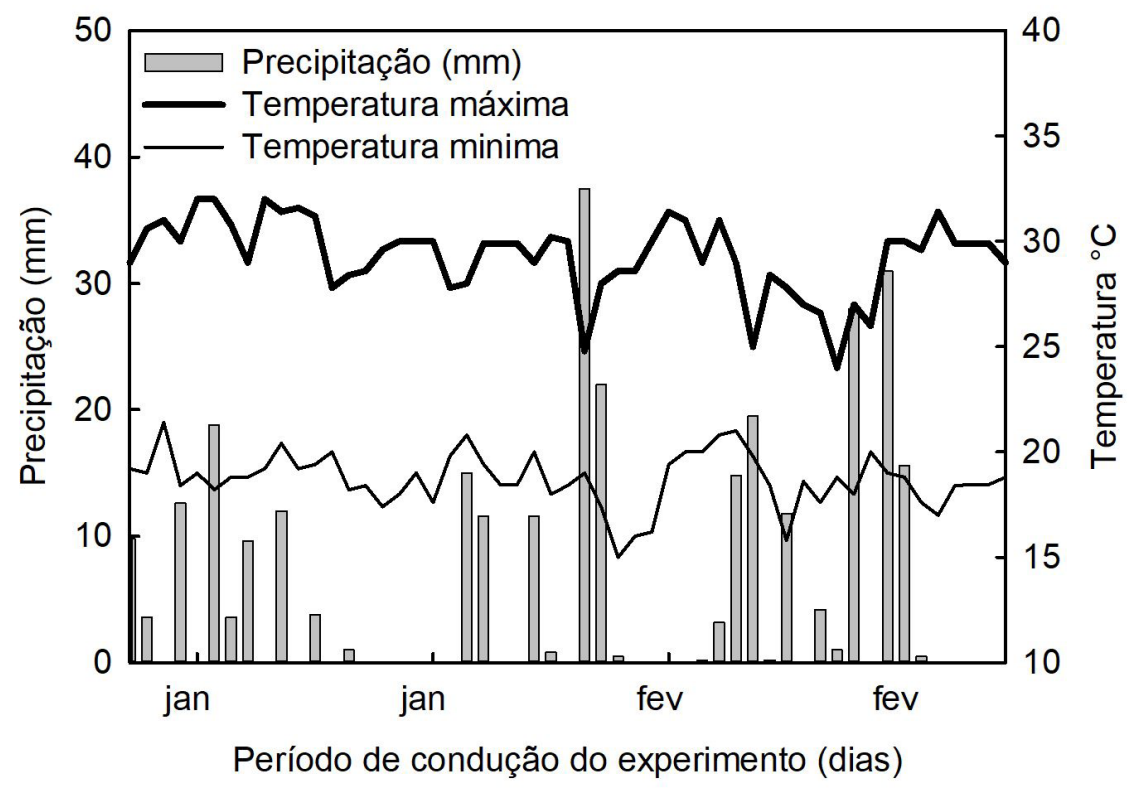

Figura 1. Precipitação, temperaturas mínima e máxima durante o período de condução do experimento. Fonte: IAPAR (Instituto Agronômico do Paraná). 
foi de $310 \mathrm{~kg} \mathrm{ha}^{-1}$ da formulação 8-20-20 (N-P-K). A aplicação dos tratamentos ocorreu quando $50 \%$ ou mais das plantas de feijoeiro começaram a emitir a terceira folha trifoliada.

Na ocasião da aplicação dos tratamentos, foi utilizado um pulverizador costal pressurizado com $\mathrm{CO}_{2}$, mantido a pressão constante de $300 \mathrm{Kpa}$, calibrado com vazão constante de $200 \mathrm{~L} \mathrm{ha}^{-1}$, com a velocidade de aplicação de $3,6 \mathrm{~km} \mathrm{~h}^{-1}$. A barra de aplicação era composta por quatro bicos espaçadas de $0,5 \mathrm{~m}$ entre si e as ponteiras de aplicação empregadas eram do tipo leque XR 110.02. As condições ambientais médias durante a aplicação foram: umidade relativa do ar $70 \%$ e temperatura $29^{\circ} \mathrm{C}$.

A tolerância relativa das plantas de feijoeiro foi avaliada aos 15, 20 e 25 dias após a aplicação (DAA). O controle de plantas de Ipomoea spp., Bidens spp. e soja foi avaliado aos 20 e 25 DAA, utilizando-se da escala visual de Frans et al. (1986) com modificações, no qual as notas foram compreendidas entre 100 (sem sintomas aparentes de injúria) e 0 (morte total das plantas). Aos 25 DAA foi avaliada a massa da parte aérea seca (MPAS) de 10 plantas de feijoeiro coletadas aleatoriamente em cada sub parcela e, a massa de plantas de Ipomoea spp., Bidens spp. e soja contidas em $1 \mathrm{~m}^{-2}$ de cada parcela.

Após a coleta para determinação da massa, foi realizada a contagem do número de plantas, o valor de massa foi dividido pelo respectivo número de plantas, resultando em massa por plantas. Os dados de MPAS de soja e infestantes foram transformados em porcentagem em relação ao tratamento sem aplicação de ethoxysulfuron. No final do ciclo da cultura foi determinado o rendimento de grãos de feijão por meio da colheita das plantas presentes na área útil de cada sub parcela $(2,6 \times 0,9 \mathrm{~m})$ e do tratamento extra sem infestantes e sem herbicida. As avaliações referentes a tolerância relativa do feijoeiro, controle e MPAS das plantas de Ipomoea spp. e Bidens spp., foram avaliadas apenas em função da parcela principal, enquanto que as avaliações referentes ao controle de soja, MPAS de soja, MPAS do feijão e o rendimento de grãos de feijão foi realizada em cada sub parcela. Para as variáveis MPAS e rendimento de grãos de feijão, procedeu-se a comparação entre os tratamentos com herbicida + infestantes e o tratamento extra sem herbicida e sem infestantes.

Os dados foram submetidos à análise da variância pelo teste $\mathrm{F}(p \leq 0,05)$ com o aplicativo computacional R (RStudio, 2016), utilizando-se do pacote ExpDes.pt (Ferreira et al., 2011). Para os dados coletados apenas nas parcelas principais se procedeu com a análise em blocos ao acaso. Para os dados coletados nas sub parcelas, a análise procedeu em blocos ao acaso com parcelas subdivididas. Quando significativos, os fatores qualitativos foram comparados pelo teste $t$ de Bonferroni (LSD protegido) $(p \leq 0,05)$. Para fatores quantitativos, quando significativos, foram realizadas regressões utilizando-se os modelos logístico de três parâmetros (Equação 1), sigmoide de quatro parâmetros (Equação 2), exponencial decrescente de três parâmetros (Equação 3) e hipérbole retangular de três parâmetros (Equação 4).

$y=a /\left[1+\left(x / D_{50}\right)^{\wedge} b\right]$

Onde: $y$ representa a resposta da variável dependente, $x$ é a dose do herbicida, $a$ é a assíntota máxima da curva, $b$ é a declividade da curva, $D_{50}$ representa a dose necessária para reduzir a variável dependente em $50 \%$.

$y=c+a /\left(1+\exp \left(-\left(x-D_{s 0}\right) / b\right)\right)$

Onde: $y, \mathrm{x}, \mathrm{e} b$ são definidos conforme já descrito anteriormente, $a$ é a máxima redução da variável dependente em função das doses de ethoxysulfuron, $c$ é a assíntota máxima da curva e $D_{50}$ com base na diferença entre a assíntota máxima e mínima da curva.

$y=c+a * \exp (-b * x)$

Onde: $y, \mathrm{x}, \mathrm{e} b$ são definidos conforme já descrito anteriormente, $a$ é a máxima redução da variável 
dependente em função das doses de ethoxysulfuron, $c$ é a assíntota mínima da curva.

$y=c+(a * x) /(b+x)$

Onde: $y$, $\mathrm{x}$, e $b$ são definidos conforme já descrito anteriormente, $a$ é o incremento máximo da variável dependente quando $x$ tende ao infinito, $c$ é a assíntota mínima da curva.

Adicionalmente foi calculado o erro padrão da média a fim de indicar a variabilidade entre as médias amostrais (Equação 5).

$E P M=\sigma / \sqrt{ } n$

Onde: $E P M$ é o erro padrão da média, $\sigma$ é o desvio padrão e $n$ é o número de amostras.

\section{Resultados e discussão}

O controle das plantas de Ipomoea spp. foi semelhante aos 20 e 25 DAA (Figura 2a), chegando a $80 \%$ com a maior dose aplicada de ethoxysulfuron (83,2 $\left.\mathrm{g} \mathrm{ha}^{-1}\right)$. Em ambos os periodos de avaliação o $D_{50}$ foi baixo (Tabela 2), sendo de 5,6 e 3,2 $\mathrm{g} \mathrm{ha}^{-1}$, para os 20 e 25 DAA respectivamente. Doses entre 0 e $10,4 \mathrm{~g} \mathrm{ha}^{-1}$ foram suficientes para reduzir $75 \%$ da massa da parte aérea seca (MPAS) das plantas de Ipomoea spp.

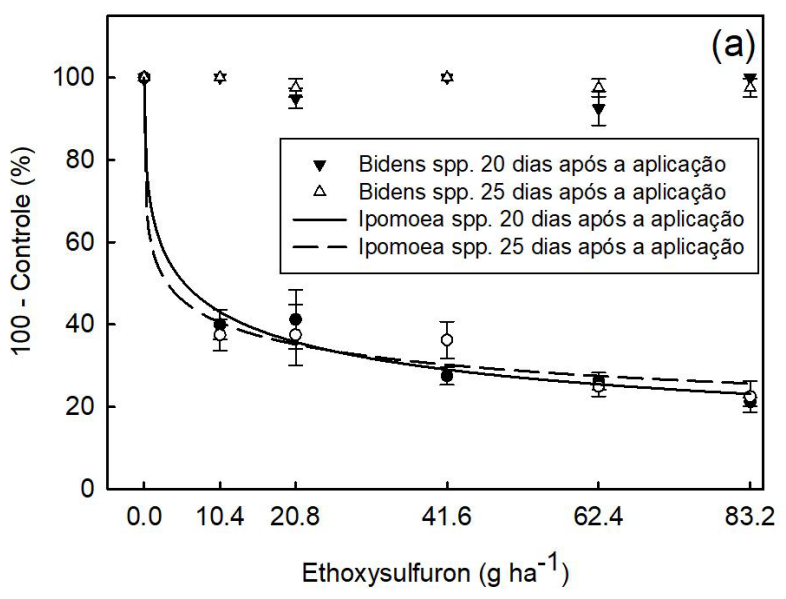

aos 25 DAA (Figura 2 b e parâmetro $c$ da Tabela 2), em doses subsequentes a MPAS manteve-se constante. Entretanto, as plantas de Bidens spp. demostraram ser insensíveis as crescentes doses de ethoxysulfuron avaliadas (Figura 2), as quais não apresentaram sintômas visuais de injúria e mantiveram a MPAS constante.

De modo geral, herbicidas inibidores da ALS são eficientes no controle de espécies pertecentes ao gênero Ipomoea. Os herbicidas do grupo químico das sulfonilureias, halosulfuron + thifensulfuron-methyl, proporcionam elevado nível de controle das plantas de Ipomoea hederifolia em trabalho apresentado anteriomente na literatura (Montgomery et al., 2015). Da mesma maneira, as imidazolinonas imazapic e imazethapyr, também proporcionam controle elevado de Ipomoea lacunosa (Grichar et al., 2012) e Ipomoea grandifolia (Vitorino e Martins, 2012).

Os inibidores da ALS também demonstram ser eficientes no controle de plantas de Bidens spp. Plantas de Bidens pilosa são facilmente controladas pelos herbicidas imazethapyr, cloransulam-methyl, chlorymuron-ethyl, nicosulfuron, metsulfuron-methyl e pyrithiobac-sodium (Christoffoleti, 2002; Lamego et al., 2009). Dentre os herbicidas citados, apenas o imazethapyr possui registro para ser

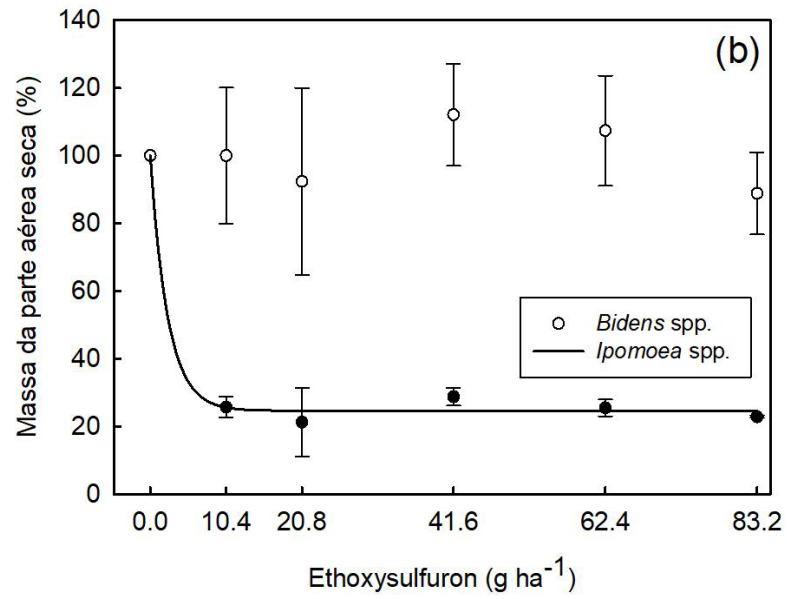

Figura 2. Efeito de ethoxysulfuron sobre: (a) controle de plantas de Ipomoea spp. e Bidens spp. aos 20 e 25 DAA; e (b) massa da parte aérea seca das plantas aos 25 DAA. Os pontos representam a média de quatro repetições e as barras correspondem o erro padrão da média. 
utilizado na cultura do feijoeiro. No entanto, quando em mistura com o fomesafen, é observado tolerância diferencial entre cultivares de feijão (Procópio et al., 2009).

Todavia, casos de resistencia de Bidens spp. a herbicidas inibidores da ALS têm sido registrados em áreas de lavoura pelo mundo, inclusive no Brasil (Takano et al., 2016). Na área em que foi conduzido o experimento é desconhecida a presença de plantas de Bidens resistentes aos herbicidas inibidores da ALS. Contudo, deve ser considerado que as doses utilizadas não foram suficientee para o controle satisfatório das plantas, a qual é acima de $100 \mathrm{~g} \mathrm{ha}^{-1}$ para algumas espécies (Rodrigues e Almeida, 2011).

Para a avaliação de controle das plantas de soja aos 20 DAA, foi constatado efeito isolado dos fatores doses de herbicida (Figura 3a e Tabela 3) e cultivares de soja (Tabela 4), enquanto que aos 25 DAA, foi observado apenas o efeito do herbicida (Figura 3a). Para a avaliação da MPAS foi observado a interação entre as doses do herbicida e as cultivares de soja (Figura 3b). Na média das três cultivares, o controle das plantas de soja (Figura 3a) foi satisfatório (80\%) aos 20 DAA, chegando a excelente $(95 \%)$ aos 25 DAA com a maior dose aplicada de ethoxysulfuron, com a

Tabela 2. Parâmetros da equação, coeficiente de determinação $\left(\mathrm{R}^{2}\right)$, quadrado médio do erro (QME) e probabilidade ( $p$ ) para as variáveis, controle de plantas de Ipomoea spp. (\%) (CI) aos 20 e 25 dias após a aplicação do ethoxysulfuron (DAA) e massa da parte aérea seca de Ipomoea spp. (MPASI) quando avaliado aos 25 DAA.

\begin{tabular}{|c|c|c|c|c|c|c|c|}
\hline \multirow{2}{*}{ Variável } & \multicolumn{4}{|c|}{ Parâmetros } & \multirow[b]{2}{*}{$\mathbf{R}^{2}$} & \multirow[b]{2}{*}{ QME } & \multirow[b]{2}{*}{$p$} \\
\hline & $a$ & $b$ & $D_{50}$ & $c$ & & & \\
\hline CI $20^{1 /}$ & $99,95(3,92)^{3 / * *}$ & $0,44(0,11)^{*}$ & $5,56(2,63)^{\mathrm{ns}}$ & - & 0,98 & 15,40 & $<0,01$ \\
\hline CI $25^{1 /}$ & $99,96(4,73)^{* *}$ & $0,33(0,13)^{\mathrm{ns}}$ & $3,18(3,09)^{\mathrm{ns}}$ & - & 0,97 & 22,35 & $<0,01$ \\
\hline MPASI $^{2 /}$ & $75,37(3,69)^{* *}$ & $0,41(0,36)^{\mathrm{ns}}$ & - & $24,63(1,66)^{* *}$ & 0,99 & 10,85 & $<0,01$ \\
\hline
\end{tabular}

${ }^{1 /}$ Logística de três parâmetros; ${ }^{2 /}$ Exponencial decrescente de três parâmetros; ${ }^{3 /}$ Valores entre parênteses indicam o erro padrão; * e ** significativo a 5 e a $1 \%$ de probabilidade.
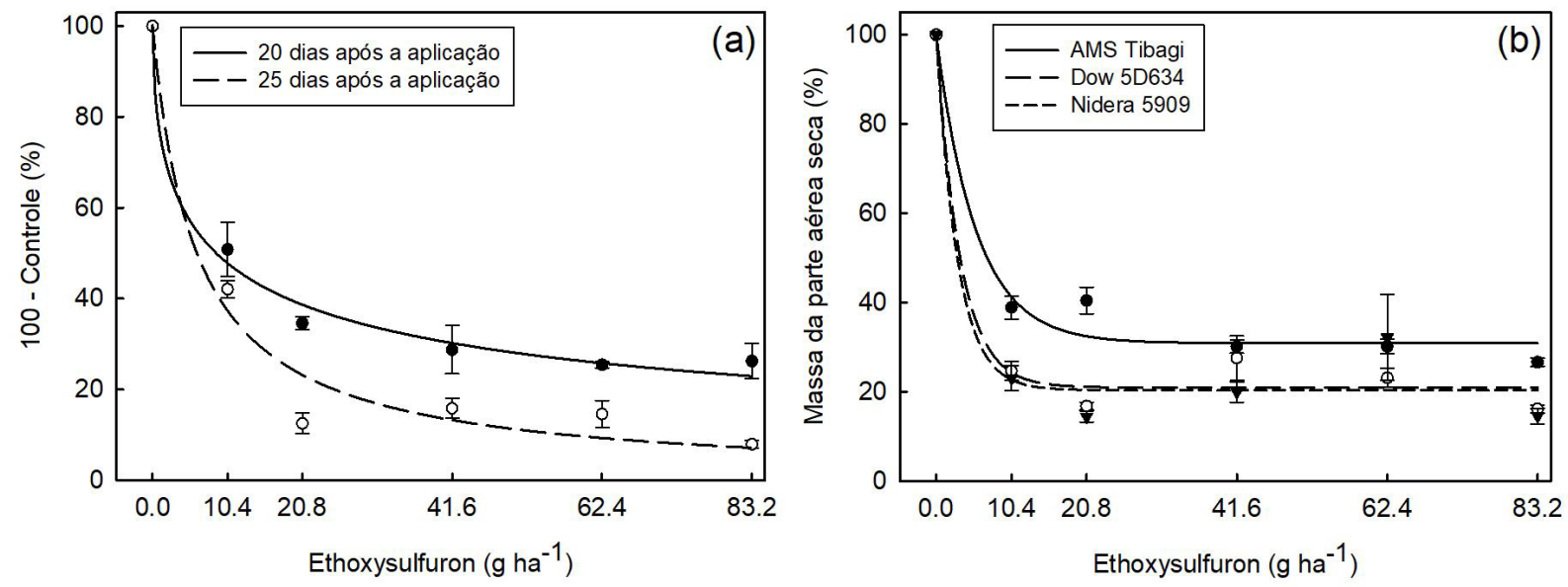

Figura 3. Efeito de ethoxysulfuron sobre: (a) o controle de plantas de soja aos 20 e 25 DAA; e (b) massa da parte aérea seca das plantas aos 25 DAA. Para a variável controle, os pontos representam a média de quatro repetições e três cultivares de soja e as barras correspondem ao erro padrão da média. Para a variável massa da parte aérea seca, os pontos representam a média de quatro repetições e as barras correspondem ao erro padrão da média. 
Tabela 3. Parâmetros da equação, coeficiente de determinação $\left(\mathrm{R}^{2}\right)$, quadrado médio do erro (QME) e probabilidade $(p)$ para as variáveis, controle de soja (\%) (CS) aos 20 e 25 DAA e massa da parte aérea seca de soja (MPASS) aos 25 DAA.

\begin{tabular}{|c|c|c|c|c|c|c|c|c|}
\hline \multirow{2}{*}{ Cultivar } & \multirow{2}{*}{ Variável } & \multicolumn{4}{|c|}{ Parâmetros } & \multirow{2}{*}{$\mathbf{R}^{2}$} & \multirow{2}{*}{ QME } & \multirow{2}{*}{$p$} \\
\hline & & $a$ & $b$ & $D_{50}$ & $c$ & & & \\
\hline & $\mathrm{CS} 20^{1 /}$ & $100,10(3,60)^{3 / * *}$ & $0,54(0,10)^{* *}$ & $8,81(2,50)^{*}$ & - & 0,98 & 12,95 & $\leq 0,01$ \\
\hline & $\operatorname{CS} 25^{1 /}$ & $100,18(7,56)^{* *}$ & $0,98(0,35)^{\mathrm{ns}}$ & $6,13(2,85)^{\mathrm{ns}}$ & - & 0,95 & 57,19 & $\leq 0,01$ \\
\hline AMS Tibagi & MPASS $25^{2 /}$ & $68,94(6,16)^{* *}$ & $0,18(0,06)^{*}$ & - & $30,88(2,93)^{* *}$ & 0,96 & 29,61 & $\leq 0,01$ \\
\hline Dow 5D634 & & $79,06(6,15)^{* *}$ & $0,30(0,18)^{\mathrm{ns}}$ & - & $20,95(2,80)^{* *}$ & 0,97 & 30,15 & $\leq 0,01$ \\
\hline Nidera 5909 & & $79,67(9,38)^{* *}$ & $0,34(0,39)^{\mathrm{ns}}$ & - & $20,34(4,24)^{*}$ & 0,94 & 70,01 & $\leq 0,01$ \\
\hline
\end{tabular}

${ }^{1 /}$ Logística de três parâmetros; ${ }^{2 /}$ Exponencial decrescente de três parâmetros; ${ }^{3 /}$ Valores entre parênteses indicam o erro padrão; * e** significativo a 5 e a $1 \%$ de probabilidade; ns= Não significativo.

Tabela 4. Valores médios de controle (\%) de três cultivares de soja aos 20 DAA de ethoxysulfuron.

\begin{tabular}{cc}
\hline Cultivar de soja & 100 - Controle (\%) \\
\hline AMS Tibagi & $48,12 \mathrm{a}^{*}$ \\
Dow 5D634 & $43,54 \mathrm{~b}$ \\
Nidera 5909 & $41,20 \mathrm{~b}$ \\
CV (\%) & 9,7 \\
\hline
\end{tabular}

*Médias seguidas pela mesma letra na mesma coluna não se diferenciam pelo Teste $t$ de Bonferroni $(p \leq 0,05)$.

$\boldsymbol{D}_{50}$ de 8,9 e $6 \mathrm{~g} \mathrm{ha}^{-1}$ para cada respectiva época de avaliação (Tabela 3). Na média das doses aos 20 DAA (Tabela 4), o controle das plantas de soja foi de aproximadamente $60 \%$ para as cultivares Dow 5D634 e Nidera 5909 e de aproximadamente $50 \%$ para a cultivar AMS Tibagi. O comportamento da MPAS aos 25 DAA(Figura 3b e Tabela 3) variou de acordo com a cultivar de soja, para as cultivares Dow 5D634 e Nidera 5909 a redução chegou a aproximadamente $80 \%$ em doses superiores a $10,4 \mathrm{~g} \mathrm{ha}^{-1}$, enquanto que para a cultivar AMS Tibagi a redução foi de aproximadamente $70 \% \mathrm{em}$ doses superiores a 20,8 $\mathrm{g} \mathrm{ha}^{-1}$.

Comumente são constatados casos de herbicidas seletivos a plantas de soja, mas não as plantas de feijão, tomando como exemplo os herbicidas chlorymuron-ethyl, cloransulam-methyl (Procópio et al., 2009) e pyroxasulfone (Soltani et al., 2009; Belfry et al., 2015a). Todavia, alguns herbicidas demostram seletividade a ambas as culturas, como é o caso do fomesafen e imazethapyr (Procópio et al., 2009). O herbicida halosulfuron que pertence ao mesmo grupo químico do ethoxysulfuron (sulfonilureia), também indica ser seletivo as plantas de feijão (Soltani et al., 2012a), entretanto não é seletivo as plantas de soja (Nandula et al., 2009). A cultivar AMS Tibagi apresentou maior restrição a imposição de controle em comparação com as demais, isto pode ocorrer devido a tolerância diferencial que ocorre entre cultivares de soja a um determinado herbicida (Miller et al., 2012; Belfry et al., 2015b).

A tolerância das plantas de feijão reduziu com o incremento das doses de ethoxysulfuron (Figura 4a,Tabela 5). Ambos aos 15 e 20 DAA a tolerância das plantas chegou a 70\% com a maior dose avaliada. Aos 25 DAA a tolerância foi de aproximadamente $90 \%$ em doses superiores a $10,4 \mathrm{~g} \mathrm{ha}^{-1}$, sendo este o período o qual as plantas apresentaram menor redução de tolerância, se comparado aos períodos de 10 e 15 DAA. Em conjunto, estes dados demonstram que as plantas de feijão possuem alta capacidade de recuperação do efeito do herbicida. Em trabalhos conduzidos avaliando o mecanismo de tolerância das plantas de feijão ao ethoxysulfuron, através do uso de inibidores da metabolização (malathion+chlorpyrifos) e protetor (mefenpyr-diethyl), constatou-se que o principal mecanismo envolvido é a metabolização da molécula do herbicida (Pagnoncelli Jr et al., 2016). 

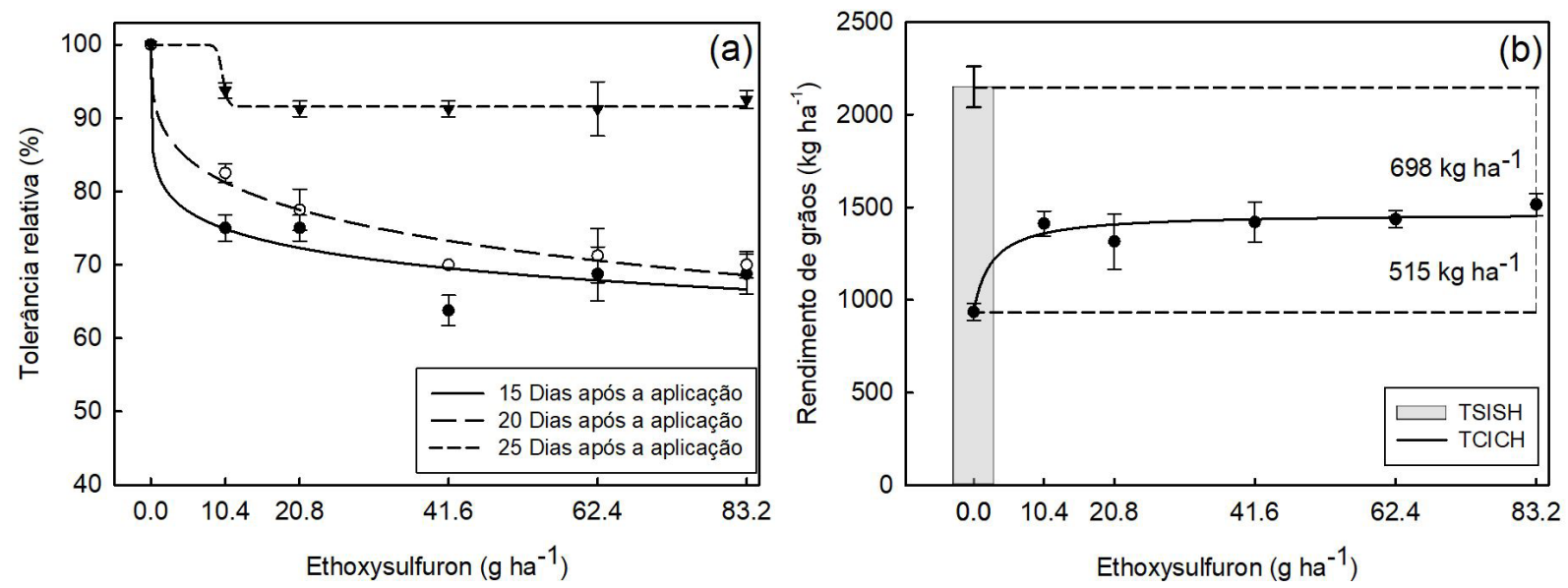

Figura 4. Efeito de ethoxysulfuron sobre: (a) tolerância relativa do feijoeiro (\%) aos 15, 20 e 25 dias após a aplicação de ethoxysulfuron (DAA); (b) rendimento de grãos $\left(\mathrm{kg} \mathrm{ha}^{-1}\right)$ em plantas de feijão cultivar IAC Imperador. Os pontos representam os valores médios de quatro repetições e as barras correspondem ao seu erro padrão. TSISH = Testemunha sem infestantes e sem a aplicação de herbicida; TCICH = Tratamento com infestante e com a aplicação do herbicida.

Para a variável MPAS das plantas de feijão, foi constatada diferenças apenas entre a testemunha sem infestantes e sem a aplicação de herbicida, se comparado com o tratamento com infestante e com a aplicação do herbicida (Tabela 6). A MPAS da testemunha sem herbicida e infestantes foi superior ao dos tratamentos com herbicida + infestantes, sendo de 13,6 e $11,3 \mathrm{~g} \mathrm{pl}^{-1}$ respectivamente. É possível verificar que em baixas doses do herbicida, a redução da MPAS ocorreu devido a competição com as infestantes na área, enquanto que em doses mais elevadas a redução ocorreu pela fitotoxicidade gerada pelo herbicida. Ademais, deve ser considerada a competição da cultura com as plantas de Bidens spp., as quais foram insensíveis em todas as faixas de doses testadas.

Para a variável rendimento de grãos observou-se efeito isolado das doses (Figura 4b), cultivares (Tabela 7) e da testemunha sem infestantes e sem a aplicação de herbicida, se comparado com o tratamento com infestante e com a aplicação do herbicida (Tabela 6). O uso do herbicida proporcionou incremento no rendimento de grãos de feijão (Figura $4 \mathrm{~b}$ e parâmetro $\boldsymbol{a}$ Tabela 5), o qual foi de aproximadamente $500 \mathrm{~kg} \mathrm{ha}^{-1} \mathrm{em}$ doses superiores a 10,4 $\mathrm{g} \mathrm{ha}^{-1}$. Entretanto, o incremento não foi suficiente para alcançar o da testemunha capinada (2272 kg ha-1), que foi superior em aproximadamente $700 \mathrm{~kg} \mathrm{ha}^{-1}$ se comparado ao rendimento proporcionado pelo tratamento com herbicida em doses superiores $10,4 \mathrm{~g} \mathrm{ha}^{-1}$ $\left(1500 \mathrm{~kg} \mathrm{ha}^{-1}\right)$. Anível de cultivar, estas demostraram dano diferenciado sobre o rendimento de grãos do feijoeiro. $\mathrm{O}$ menor rendimento de grãos foi observado quando as plantas de feijão competiram com a cultivar AMS Tibagi (1261 kg ha-1), em comparação as plantas das cultivares Dow 5D634 $\left(1417 \mathrm{~kg} \mathrm{ha}^{-1}\right)$ e Nidera 5909 (1459 kg ha-1) (Tabela 7). Esta diferença pode ser atribuída a menor redução de MPAS gerada pelo herbicida sobre a cultivar AMS Tibagi em comparação as demais cultivares. O rendimento de grãos da testemunha sem herbicida e infestantes (Tabela 6) foi superior em aproximadamente $900 \mathrm{~kg} \mathrm{ha}^{-1}$, se comparado com o tratamento com herbicida + infestantes.

De maneira geral, a faixa de doses que compreende entre 0 e 20,8 $\mathrm{g} \mathrm{ha}^{-1}$, indicam ser suficientes para proporcionar níveis de controle e redução da MPAS das plantas de Ipomoea spp. e soja a níveis 
Tabela 5. Parâmetros da equação, coeficiente de determinação $\left(\mathrm{R}^{2}\right)$, quadrado médio do erro (QME) e probabilidade $(p)$ para as variáveis, tolerância relativa do feijoeiro (\%) (TR) aos 15, 20 e 25 dias após a aplicação do ethoxysulfuron (DAA) e rendimento de grãos de feijão $\left(\mathrm{kg} \mathrm{ha}^{-1}\right)$.

\begin{tabular}{|c|c|c|c|c|c|c|c|}
\hline \multirow{2}{*}{ Variável } & \multicolumn{4}{|c|}{ Parâmetros } & \multirow{2}{*}{$\mathbf{R}^{2}$} & \multirow{2}{*}{ QME } & \multirow[b]{2}{*}{$p$} \\
\hline & $a$ & $b$ & $D_{50}$ & $c$ & & & \\
\hline TR $15^{1 /}$ & $100,02(3,92)^{4 / * *}$ & $0,19(0,12)^{\mathrm{ns}}$ & $3004,94(8007,37)^{\mathrm{ns}}$ & - & 0,90 & 15,37 & 0,01 \\
\hline TR $20^{1 /}$ & $100,09(2,22)^{* *}$ & $0,33(0,07)^{*}$ & $888,36(637,66)^{\mathrm{ns}}$ & - & 0,96 & 4,93 & 0,003 \\
\hline TR $25^{2 /}$ & $-8,44(1,12)^{*}$ & $0,36(1,60)^{\mathrm{ns}}$ & $10,02(3,41)^{\mathrm{ns}}$ & $100,00(1,8$ & 0,95 & 0,58 & 0,03 \\
\hline Rendimento $^{3 /}$ & $531,00(93,02)^{*}$ & - & $2,64(3,02)^{*}$ & $936,85(72,01)^{* *}$ & 0,88 & 5186,00 & 0,02 \\
\hline
\end{tabular}

${ }^{1 /}$ Logística de três parâmetros; ${ }^{2 /}$ Sigmoide de quatro parâmetros; $;{ }^{3 /}$ Hipérbole retangular de três parâmetros; ${ }^{4 /}$ Valores entre parênteses indicam o erro padrão; ${ }^{*} \mathrm{e} * *$ significativo a 5 e a $1 \%$ de probabilidade; ${ }^{\text {ns }}$ Não significativo.

Tabela 6. Teste de contraste entre a testemunha sem infestantes e sem a aplicação de herbicida (TSISH) e o tratamento com infestante e com a aplicação do herbicida (TCICH) sobre a MPAS das plantas de feijão $\left(\mathrm{g} \mathrm{pl}^{-1}\right)$ e o rendimento de grãos $\left(\mathrm{kg} \mathrm{ha}^{-1}\right)$ da cultura.

\begin{tabular}{ccc}
\hline \multirow{2}{*}{ Variável } & \multicolumn{1}{c}{ MPAS } & Rendimento de grãos \\
\cline { 2 - 3 } $\mathbf{g ~ p l}^{-1}$ & $\mathbf{k g ~ h a}^{-1}$ \\
\hline TSISH & $13,6 \mathrm{a}^{*}$ & $2272,44 \mathrm{a}$ \\
TCICH & $11,3 \mathrm{~b}$ & $1380,62 \mathrm{~b}$ \\
CV $(\%)$ & 14,6 & 17,6 \\
\hline
\end{tabular}

*Médias seguidas pela mesma letra na mesma coluna não se diferenciam pelo Teste $t$ de Bonferroni $(p \leq 0,05)$.

Tabela 7. Valores médios do rendimento de grãos $\left(\mathrm{kg} \mathrm{ha}^{-1}\right)$ de feijão quando submetido a competição com três cultivares de soja durante todo o ciclo de desenvolvimento.

\begin{tabular}{cc}
\hline Cultivar de soja & Rendimento de grãos kg hä \\
\hline AMS Tibagi & $1260,93 \mathrm{~b} *$ \\
Dow 5D634 & $1417,36 \mathrm{ab}$ \\
Nidera 5909 & $1458,75 \mathrm{a}$ \\
CV (\%) & 17,6 \\
\hline
\end{tabular}

*Médias seguidas pela mesma letra na mesma coluna não se diferenciam pelo Teste $\mathrm{t}$ de Bonferroni $(p \leq 0,05)$.

satisfatórios $(>80 \%)$. Doses compreendidas dentro desta faixa foram suficientes para incrementar o rendimento de grãos das plantas de feijão. Porém, em doses superiores, apesar de o herbicida controlar satisfatoriamente as plantas de Ipomoea spp. e soja, a competição com as plantas de Bidens spp. e a injúria gerada pelo herbicida, resultaram na estagnação do incremento do rendimento de grãos. Conforme demonstrado por Galon et al. (2016), quando a densidade de plantas de Bidens spp. é de $10 \mathrm{pl} \mathrm{m}^{-2}$, a perda de rendimento do feijoeiro pode chegar a $35 \%$.

Em algumas regiões brasileiras, tem se tornado comum a prática do cultivo feijão em sucessão ao da soja. Dentre as consequências, destaca-se a dificuldade do manejo das plantas de soja remanescentes na área, que além de atuarem como competidoras diretas por recursos, dificultam a aceitação do produto devido à dificuldade da separação dos grãos de feijão dos de soja. Devido principalmente ao potencial uso do herbicida para o controle da soja tiguera, mais estudos avaliando o efeito do ethoxysulfuron sobre a cultura do feijão deveriam ser conduzidos, principalmente no que se refere a associação com outros latifolicidas já registrados para o feijoeiro. Conforme demonstrado por Soltani et al. (2012b), a associação de bentazon com diferentes herbicidas inibidores da ALS, auxilia na redução da injúria proporcionada por estes últimos sobre a cultura do feijão.

\section{Conclusões}

Doses de até 20,8 $\mathrm{g} \mathrm{ha}^{-1}$ são suficientes para controlar satisfatoriamente plantas de Ipomoea spp.

O herbicida proporcionou incremento no rendimento de grãos de feijão, todavia, não foi suficiente para alcançar o rendimento da testemunha livre da interferência de plantas daninhas. 
A cultivar de feijoeiro IAC Imperador demostrou tolerância moderada ao ethoxysulfuron, observando tendência de rápida recuperação da injúria do herbicida.

\section{Referências}

Assis, A.C.L.P.; Reis, M.R.; Pessoa, G.D.O.; Silva, D.V.; Hayata, M.; Dias, R.C.; Rocha, B.H. Seletividade do ethoxysulfuron às culturas da soja e feijão. Revista Brasileira de Herbicidas, v.13, n.2, p.117-124, 2014.

Belfry, K.D.; Mcnaughton, K.E.; Sikkema, P.H. Weed control in soybean using pyroxasulfone and sulfentrazone. Canadian Journal of Plant Science, v.95, n.6, p.1199-1204, 2015a.

Belfry, K.D.; Soltani, N.; Brown, L.R.; Sikkema, P.H. Tolerance of identity preserved soybean cultivars to preemergence herbicides. Canadian Journal of Plant Science, v.95, n.4, p.719-726, 2015 b.

Borchartt, L.; Jakelaitis, A.; Valadão, F.C.A.; Venturoso, L.A.C.; Santos, C.L. Períodos de interferência de plantas daninhas na cultura do feijoeiro-comum (Phaseolus vulgaris L.). Revista Ciência Agronômica, v.42, n.3, p.725-734, 2011.

Brasil. Ministério da Agricultura Pecuária e Abastecimento. Consulta de ingrediente ativo. Brasília: MAPA, 2016. Disponível em: <http:// extranet.agricultura.gov.br>. Acesso em: 19 out. 2016

Chiorato, A.F.; Carbonell, S.A.M.; Carvalho, C.R.L.; Barros, V.L.N.P.; Borges, W.L.B.; Ticelli, M.; et al. 'IAC IMPERADOR': early maturity "carioca" bean cultivar. Crop Breeding and Applied Biotechnology, v.12, n.4, p.297-300, 2012.

Christoffoleti, P.J. Curvas de dose-resposta de biótipos resistente e suscetível de Bidens pilosa L. aos herbicidas inibidores da ALS. Scientia Agrícola, v.59, n.3, p.513-519, 2002.
Ferreira, E.B.; Cavalcanti, P.P.; Nogueira, D.A. ExpDes: experimental designs package R package version 1.1.1. Vienna: R Foundation for Statistical Computing, 2011. Disponível em: $<$ https://cran.rproject.org/web/packages/ExpDes.pt/ExpDes. pt.pdf $>$. Acesso em: 19 out. 2016.

Frans, R.; Talbot, R.; Marx, D.; Crowley, H. Experimental design and techniques for measuring and analysing plant responses to weed control practices. In: Camper, N.D. Research methods in weed science. 3 th ed. Champaign: SWSS, 1986. p.29-46.

Galon, L.; Forte, C.T.; Gabiatti, R.L.; Radunz, L.L.; Aspiazú, I.; Kujawinski, R.; et al. Interference and economic threshold level for control of beggartick on bean cultivars. Planta Daninha, v.34, n.3, p.411-422, 2016.

Grichar, W.J.; Jordan, D.L.; Prostko, E.P. Weed control and peanut (Arachis hypogaea L.) response to formulations of imazapic. Crop Protection, v.36, p.31-36, 2012.

Kozlowski, L.A.; Ronzelli Júnior, P.; Purissimo, C.; Daros, E.; Koehler, H.S. Período crítico de interferência das plantas daninhas na cultura do feijoeiro-comum em sistema de semeadura direta. Planta Daninha, v.20, n.2, p.213-220, 2002.

Lamego, F.P.; Vidal, R.A.; Burgos, N.R.; Federizzi, L.C. Cross-resistance of Bidens subalternans to acetolactate synthase inhibitors in Brazil. Weed Research, v.49, n.6, p.634-641, 2009.

Miller, R.T.; Soltani, N.; Robinson, D.E.; Kraus, T.E.; Sikkema, P.H. Soybean (Glycine max) cultivar tolerance to saflufenacil. Canadian Journal of Plant Science, v.92, n.7, p.1319-1328, 2012.

Montgomery, G.B.; Bond, J.A.; Golden, G.R.; Gore, J.; Edwards, M.; Eubank, T.W.; Walker, T.W. Utilization of Saflufenacil in a Clearfield ${ }^{\circledR}$ Rice (Oryza sativa) System. Weed Technology, v.29, n.2, p.255-262, 2015.

Nandula, V.K.; Poston, D.H.; Reddy, K.N.; Whiting, K. Response of Soybean to Halosulfuron 
Herbicide. International Journal of Agronomy, v.2009, p. 754510, 2009.

Oliveira Junior, R.S.; Inoue, M.H. Seletividade de herbicidas para culturas e plantas daninhas. In: Oliveira Junior, R.S.; Constantin, J.; Inoue, M.H. Biologia e manejo de plantas daninhas. Curitiba: Omnipax, 2011. 348p.

Pagnoncelli Jr, F.D.B.; Vidal, R.A.; Trezzi, M.M.; Machado, A.; Gallon, M.; Xavier, E.; Oliveira, M.C. Tolerance of common bean plants to ethoxysulfuron herbicide and the mechanism involved in the process. Planta Daninha, v.34, n.3, p.535-543, 2016.

Procopio, S.O.; Braz, A.J.B.P.; Barroso, A.L.L.; Cargnelutti Filho, A.; Cruvinel, K.L.; Betta, M.; et al. Potencial de uso dos herbicidas chlorimuron-ethyl, imazethapyr e cloransulam-methyl na cultura do feijão. Planta Daninha, v.27, n.2, p.327-336, 2009.

Radosevich, S.R.; Holt, J.S.; Ghersa, C.M. Ecology of weeds and invasive plants: relationship to agriculture and natural resource management. New York: Wiley, 2007. 475p.

Rodrigues, B.N.; Almeida, F.S. Guia de herbicidas. 6. ed. Londrina: Autores, 2011. 268p.

RStudio Team. Integrated development for $\mathbf{R}$. Boston: RStudio, 2016. Disponivel em: $<\mathrm{http}: / /$ www.rstudio.com/>. Acesso em: 19 out. 2016.
Soltani, N.; Shropshire, C.; Sikkema, P.H. Response of dry bean to pre plant incorporated and pre emergence applications of pyroxasulfone. Canadian Journal of Plant Science, v.89, n.5, p.993-997, 2009.

Soltani, N.; Shropshire, C.; Sikkema, P.H. Response of dry beans to halosulfuron applied postemergence. Canadian Journal of Plant Science, v.92, n.4, p.723-728, 2012a.

Soltani, N.; Shropshire, C.; Sikkema, P.H. Safening effect of bentazon on cloransulam-methyl and halosulfuron-methyl in dry bean. Agricultural Science, v.3, n.3, p.368-374, 2012 b.

Takano, H.K.; Oliveira Junior, R.S.; Constantin, J.; Braz, G.B.P.; Franchini, L.H.M.; Burgos, N.R. Multiple resistance to atrazine and imazethapyr in hairy beggarticks (Bidens pilosa). Ciência e Agrotecnologia, v.40, n.5, p-547-554. 2016.

Vitorino, H.S.; Martins, D. Efeito do déficit hídrico na eficiência de herbicidas e nas características bioquímicas de Ipomoea grandifolia. Planta Daninha, v.30, n.1, p.185-191, 2012.

Wassmuth, B.E.; Stoll, P.; Tscharntke, T.; Thies, C. Spatial aggregation facilitates coexistence and diversity of wild plant species in field margins. Perspectives in Plant Ecology, Evolution and Systematics, v.11, n.2, p.127-135, 2009. 